\title{
Quantification of Foot-and-mouth Disease Virus Transmission Rates Using Published Data
}

\author{
Nesya E. Goris ${ }^{1}$, Phaedra L. Eblé ${ }^{2}$, Mart C. M. de Jong ${ }^{3}$ and Kris De Clercq ${ }^{1}$ \\ ${ }^{1}$ Epizootic Diseases Section, Department of Virology, Veterinary and Agrochemical Research Centre, Brussels, Belgium; \\ ${ }^{2}$ Central Veterinary Institute of Wageningen UR, Lelystad, The Netherlands; ${ }^{3}$ Quantitative Veterinary Epidemiology group, \\ Wageningen University and Research Centre, Wageningen, The Netherlands
}

\begin{abstract}
Summary
Foot-and-mouth disease is an extremely infectious and devastating disease affecting all species of clovenhoofed animals. To understand the epidemiology of the causative virus and predict viral transmission dynamics, quantified transmission parameters are essential to decision makers and modellers alike. However, such quantified parameters are scarcely available, and recently a series of animal experiments was set up to obtain such data experimentally. In this communication, however, we report on the use of data from an animal experiment conducted 10 years ago to quantify transmission of foot-and-mouth disease virus between non-vaccinated sheep and from sub-clinically infected sheep to in-contact pigs. This new analysis utilises a state-of-the-art Generalised Linear Model to estimate the transmission rate. From the obtained results it is concluded that meta-analysis of "old" experiments using newly developed techniques can provide useful data to replace, reduce and refine future foot-and-mouth disease transmission experiments, thereby minimising animal suffering for research purposes.
\end{abstract}

Keywords: foot-and-mouth disease, transmission, sheep, pigs, $3 R$

\section{Introduction}

Foot-and-mouth disease (FMD) is an extremely infectious and devastating disease (Goris et al., 2008). The causative virus affects all members of the Artiodactyla, among which are economically important species such as cattle, pigs, sheep and goats. The role of sheep in the transmission of the FMD virus (FMDV) is somewhat controversial. Nevertheless, Barnett and Cox (1999) concluded that there is much circumstantial evidence to suggest that small ruminants may be important in the natural epidemiology of FMDV. Moreover, since FMDV causes milder, usually sub-clinical disease in sheep (Kitching and Hughes, 2002), FMD can go undetected for a relatively long period of time as exemplified by the 2001 FMD epidemic in the United Kingdom. To predict the impact of control measures on viral spread during a FMD outbreak, transmission parameters are essential to modellers and decision makers. The absence of quantified transmission parameters in sheep was the basis for recent FMDV transmission experiments between lambs housed in the same stable (Orsel et al., 2007). In nonvaccinated lambs, the transmission rate $\beta$, which is defined as the average number of secondary infections per unit of time per infected animal, was estimated to be 0.105 per day [95\% confidence interval (CI): $0.044-0.253$ ]. The associated reproduction ratio $R$ (i.e. the average number of secondary infections per infectious individual during its entire infectious period) of 2.22 [95\% CI: 0.46-4.33] indicates that one infected sheep can be the source of minor and major FMDV outbreaks within a susceptible sheep population (Orsel et al., 2007).

Although this information is valuable, additional data on the spread of FMDV from sheep to other species - and especially to pigs which have the ability upon infection to excrete huge amounts of airborne virus (Alexandersen and Donaldson, 2002) - is also urgently needed. However, in light of the $3 \mathrm{R}$ principle (Replacement, Reduction and Refinement) on animal use and before designing new transmission experiments and thus subjecting animals to FMDV infection, it is advisable to analyse data already available from past animal 
trials with state-of-the-art mathematical models and statistical techniques. Thus, data of a study conducted 10 years ago in sheep and pigs to examine the relative sensitivity of virus isolation (VI) and of a reverse-transcription polymerase chain reaction test followed by digoxigenin ELISA detection (RTPCR-ELISA) (Callens et al., 1998), was re-analysed in terms of FMDV transmission using the statistical analysis based on the stochastic SIR model (susceptible-infectious-recovered) described by de Jong and Kimman (1994).

\section{Experimental design and results}

Briefly, two non-vaccinated sheep were experimentally infected in the tongue epithelium, intranasally and intradermally with FMDV $\mathrm{O}_{1}$ Romania at $10^{7} \mathrm{ID}_{50}$ (i.e. $50 \%$ infective dose). One day later four non-vaccinated sheep were brought into contact with them. The infected sheep and contact sheep were allowed to mingle freely and various samples (i.e. serum, nose and mouth swabs, faeces, wool, milk, spleen, kidney, ganglion, stomach, etc.) were taken over a period of at least one month for serological (antibody detection ELISA) and virological assessment (VI and RT-PCR-ELISA). Although the contact sheep showed no FMDV-induced clinical signs, sub-clinical FMDV infection was clearly demonstrated later following serological and virological examination. Twenty-four days post contact (dpc) two non-vaccinated sentinel pigs were put into contact with three remaining contact-infected sheep. The inoculated and other contact sheep had already died from FMDV infection or were slaughtered previously to assess the relative sensitivity of the different tests. RTPCR-ELISA positive results on serum samples were obtained for both sentinel pigs at 12 and $14 \mathrm{dpc}$. For more detailed information on the experimental design and the results, the reader is referred to Callens et al. (1998).

VI and RT-PCR-ELISA results on nose and mouth swabs and on sera were used to estimate $\beta$ between non-vaccinated sheep and from contact-infected sheep to non-vaccinated sentinel pigs by applying the Generalised Linear Model (GLM) as described by Eblé et al. (2008). The GLM was based on a stochastic S(usceptible) I(nfectious) R(emoved) model in which transmission is described by the change in number of $\mathrm{S}, \mathrm{I}, \mathrm{R}$ and the total number of animals. The results for within-sheep transmission based on VI results predicted a value for $\beta$ of $0.60 \mathrm{day}^{-1}$ [95\% CI: $0.23-1.61$ ], whereas a value of 1.66 day $^{-1}$ [95\% CI: 0.60-4.59] was recorded for within-sheep transmission based on RT-PCR-ELISA results. Furthermore, $\beta$ was found to be 0.24 per day [95\% CI: 0.06-0.98] for FMDV transmission from contact-infected sheep to sentinel pigs within the same pen.

The infectious period $\mathrm{T}$ however, could not be estimated since all animals were slaughtered before clearing FMDV. Hence, R cannot be inferred, but could be deduced from similar experiments even if differences in challenge conditions may lead to a variation in $\mathrm{T}$.

\section{Discussion}

Although the statistical power of the above-mentioned experiment is relatively low given the limited numbers of animal used, two conclusions can nonetheless be drawn from this analysis. First of all, FMDV transmission within a group of free-mingling sheep can occur, which confirms previously published data (Orsel et al., 2007). On average and depending on the sensitivity and nature of the detection assay (e.g. live virus detection using VI versus viral RNA measurement using RT-PCR-ELISA), 0.60 [95\% CI: 0.23-1.61] to 1.66 [95\% CI: 0.60-4.59] newly infected cases arise per day per infected animal. It should, however, be noted that these estimates are considerably higher than the previously reported 0.11 day $^{-1}$ [95\%: 0.04-0.25] for FMDV O/NET/2001 (Orsel et al., 2007). Differences in the virulence of the FMDV strains, in the infection route, the infective dose, etc. are certainly at the origin of some of the observed variation. It is, therefore, strongly advised to design animal transmission experiments that mimic natural in-field conditions as closely as possible. For instance, in this communication the experimentally infected sheep were most likely over-challenged resulting in a higher estimate of $\beta$.

Secondly, transmission from sub-clinically infected sheep at 24 days post infection to in-contact pigs is possible, particularly when evidence of viral RNA presence in nasal and mouth excretions has been demonstrated (e.g. by means of RT-PCR-ELISA). However, the rate of transmission is reduced to 0.24 new cases per day [95\% CI: 0.06-0.98], which to the best of our knowledge is the first reported quantified transmission parameter from sub-clinically-infected sheep to in-contact pigs. Even though a relatively low number of newly infected pigs would result from direct contact with subclinically-infected sheep, the presence of one infected pig in a susceptible herd poses a great risk, especially to mixed livestock herds and neighbouring farms, as huge amounts of FMDV are excreted by this species (Alexandersen and Donaldson, 2002).

Many in vivo experiments using FMDV have been and are being conducted to gather data on diagnostic test performances, on the ability of conventional and new generation vaccines to confer protection or reduce viral excretion, to study the efficacy of certain antiviral compounds, etc. As demonstrated in this communication, analysis of these "old" data may prove valuable to quantify FMDV transmission parameters both within and between susceptible species and may help answer some basic questions concerning the epidemiology and spread of FMDV, thereby reducing and/or replacing reliance on further animal testing. Moreover, grouping these data and performing a meta-analysis would increase the statistical power of the stochastic model (Eblé et al., 2008). In any case, it could provide initial estimates of transmission and be the basis for the refined design of future FMDV in vivo transmission experiments. 


\section{References}

Alexandersen, S. and Donaldson, A. I. (2002). Further studies to quantify the dose of natural aerosols of foot-and-mouth disease virus for pigs. Epidemiol. Infect. 128, 313-323.

Barnett, P. V. and Cox, S. J. (1999). The role of small ruminants in the epidemiology and transmission of foot-and-mouth disease. Vet. J. 158, 6-13.

Callens, M., De Clercq, K., Gruia, M. and Danes, M. (1998). Detection of foot-and-mouth disease by reverse transcription polymerase chain reaction and virus isolation in contact sheep without clinical signs of foot-and-mouth disease. Vet. Q. 20(S2), 37-40.

de Jong, M. C. and Kimman, T. G. (1994). Experimental quantification of vaccine-induced reduction in virus transmission. Vaccine 12, 761-766.

Eblé, P. L., de Koeijer, A. A., de Jong, M. C. M. et al. (2008). A meta-analysis quantifying transmission parameters of FMDV strain $\mathrm{O}$ Taiwan among non-vaccinated and vaccinated pigs. Prev. Vet. Med. 83, 98-106.

Goris, N., Vandenbussche, F. and De Clercq, K. (2008). Potential of antiviral therapy and prophylaxis for controlling RNA viral infections of livestock. Antiviral Res. 78, 170-178.

Kitching, R. P. and Hughes, G. J. (2002). Clinical variation in foot and mouth disease: sheep and goats. Rev. Sci. Tech. 21, 505-512.

Orsel, K., Dekker, A., Bouma, A. et al. (2007). Quantification of foot and mouth disease virus excretion and transmission within groups of lambs with and without vaccination. Vaccine $25,2673-2679$.

\section{Acknowledgements}

The work was supported by the sixth framework programme EPIZONE (FOOD-CT-2006-016236).

\section{Correspondence to}

Dr. Nesya Goris

Veterinary and Agrochemical Research Centre

Virology Department

Epizootic Diseases Section

Groeselenberg 99

1180 Brussels

Belgium

e-mail: negor@var.fgov.be

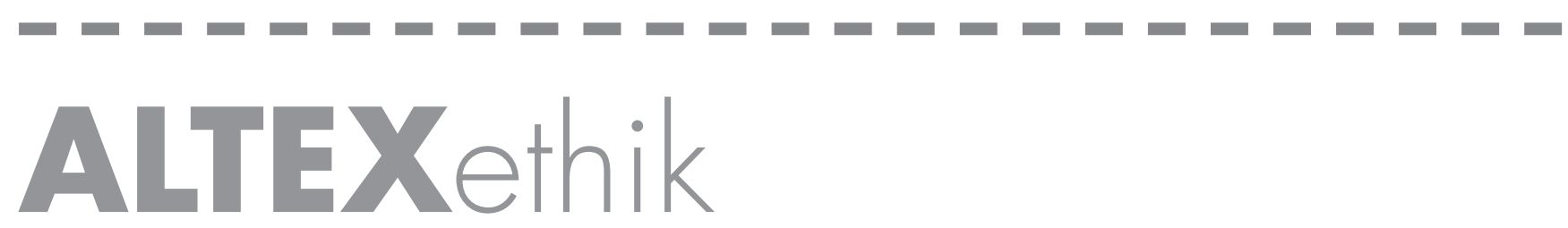

Would you like to receive ALTEXethik?

Dear subscriber,

your current subscription entitles you to four issues of ALTEX per year and, if a sponsor agrees to pay the printing and shipping costs, you will also receive the supplements for e.g. the Linz conference or the World Congress on Alternatives.

The new, additional issue ALTEXethik, which shall contain articles about the relationship between humans and animals, the literature report and summaries of all full articles published in ALTEX during the year - all written in German - is not included in the normal subscription but costs an additional $€ 10$ per year.

To subscribe to ALTEXethik, please fill in this page and fax it to +49753125833 or +41443800832 , or send an e-mail with the relevant information to abo@altex.ch

Yes, please add a subscription to ALTEXethik to my normal subscription for only $10 €$ extra (15 $€$ outside Europe), shipping included.

Name:

Address:

Signature: 